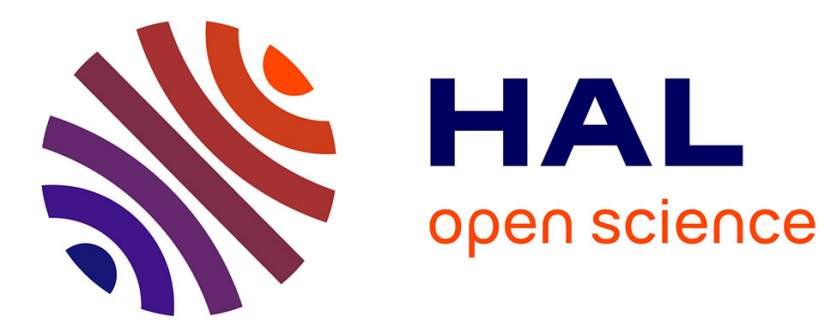

\title{
Uniqueness and stability in an inverse problem for the Schrödinger equation
}

Lucie Baudouin, Jean-Pierre Puel

\section{To cite this version:}

Lucie Baudouin, Jean-Pierre Puel. Uniqueness and stability in an inverse problem for the Schrödinger equation. Inverse Problems, 2002, 18 (6), pp.1537-1554. hal-00143868

\section{HAL Id: hal-00143868 \\ https://hal.science/hal-00143868}

Submitted on 2 May 2007

HAL is a multi-disciplinary open access archive for the deposit and dissemination of scientific research documents, whether they are published or not. The documents may come from teaching and research institutions in France or abroad, or from public or private research centers.
L'archive ouverte pluridisciplinaire HAL, est destinée au dépôt et à la diffusion de documents scientifiques de niveau recherche, publiés ou non, émanant des établissements d'enseignement et de recherche français ou étrangers, des laboratoires publics ou privés. 


\title{
UNIQUENESS AND STABILITY IN AN INVERSE PROBLEM FOR THE SCHRÖDINGER EQUATION.
}

\author{
Lucie BAUdouIN Jean-Pierre PUEL *
}

\begin{abstract}
We study the Schrödinger equation $i y^{\prime}+\Delta y+q y=0$ in $\Omega \times(0, T)$ with Dirichlet boundary data $\left.y\right|_{\partial \Omega \times(0, T)}$ and real valued initial condition $\left.y\right|_{\Omega \times\{0\}}$ and we consider the inverse problem of determining the potential $q(x), x \in \Omega$ when $\left.\frac{\partial y}{\partial \nu}\right|_{\Gamma_{0} \times(0, T)}$ is given. Here $\Omega$ is an open bounded domain of $\mathbb{R}^{N}, \Gamma_{0}$ is an open subset of $\partial \Omega$ satisfying a suitable geometrical condition and $T>0$. More precisely, from a global Carleman estimate we prove a stability inequality between $\|p-q\|$ and $\left\|\frac{\partial y(q)}{\partial \nu}-\frac{\partial y(p)}{\partial \nu}\right\|$ with appropriate norms.
\end{abstract}

Keywords: Inverse problem, Schrödinger equation, Dirichlet boundary conditions.

AMS Classification: 35R30, 31B20

\section{Introduction}

Let $N \in \mathbb{N}, T>0$ and let $\Omega \subset \mathbb{R}^{N}$ be a bounded domain with $C^{2}$-boundary $\partial \Omega$. Let $\Gamma_{0}$ be an open subset of $\partial \Omega$.

Throughout this paper, we use the following notations :

$$
\begin{aligned}
& \nabla v=\left(\frac{\partial v}{\partial x_{1}}, \ldots, \frac{\partial v}{\partial x_{N}}\right), D^{2} v=\left(\frac{\partial^{2} v}{\partial x_{i} \partial x_{j}}\right)_{1 \leq i, j \leq N}, \\
& \Delta v=\sum_{i=1}^{N} \frac{\partial^{2} v}{\partial x_{i}^{2}}, v^{\prime}=\frac{\partial v}{\partial t} \text { and } v^{\prime \prime}=\frac{\partial^{2} v}{\partial t^{2}}, \\
& \nu \in \mathbb{R}^{N} \text { denotes the unit outward normal vector to } \partial \Omega, \\
& \frac{\partial v}{\partial \nu}=\nabla v \cdot \nu \text { is the normal derivative. }
\end{aligned}
$$

*baudouin@math.uvsq.fr, jppuel@cmapx.polytechnique.fr, Laboratoire de Mathématiques Appliquées, Université de Versailles Saint-Quentin, 45 avenue des Etats Unis, 78035 Versailles Cedex, France. 
We consider the Schrödinger equation :

$$
\left\{\begin{array}{l}
i y^{\prime}(x, t)+\Delta y(x, t)+q(x) y(x, t)=0, x \in \Omega, t \in(0, T) \\
y(x, t)=h(x, t), x \in \partial \Omega, t \in(0, T) \\
y(x, 0)=y_{0}(x), x \in \Omega .
\end{array}\right.
$$

This paper treats two kinds of inverse problems which can be stated as follows.

Non linear inverse Problem : Is it possible to retrieve the potential $q=q(x), x \in$ $\Omega$ from measurement of the normal derivative

$$
\left.\frac{\partial y}{\partial \nu}\right|_{\Gamma_{0} \times(0, T)}
$$

where $y$ is the solution to (1)?

In this direction, we will answer to two more precise problems.

Uniqueness : Under geometrical conditions on $\Gamma_{0}$, does the equality $\frac{\partial y(q)}{\partial \nu}=\frac{\partial y(p)}{\partial \nu}$ on $\Gamma_{0} \times(0, T)$ imply $q=p$ on $\Omega$ ?

Stability : Under geometrical conditions on $\Gamma_{0}$, is it possible to estimate $\|q-p\|_{L^{2}(\Omega)}$ or better, a stronger norm of $(p-q)$, by a suitable norm of

$$
\left.\left(\frac{\partial y(q)}{\partial \nu}-\frac{\partial y(p)}{\partial \nu}\right)\right|_{\Gamma_{0} \times(0, T)} ?
$$

Indeed, we will only give a local answer about the determination of $q$. We will first work on a linearized version of the problem and consider the following Schrödinger equation :

$$
\left\{\begin{array}{l}
i u^{\prime}(x, t)+\Delta u(x, t)+q(x) u(x, t)=f(x) R(x, t), x \in \Omega, t \in(0, T) \\
u(x, t)=0, x \in \partial \Omega, t \in(0, T) \\
u(x, 0)=0, x \in \Omega
\end{array}\right.
$$

Linear inverse problem : Is it possible to determine $f(x), x \in \Omega$ from the knowledge of the normal derivative

$$
\left.\frac{\partial u}{\partial \nu}\right|_{\Gamma_{0} \times(0, T)}
$$

where $R$ and $q$ are given and $u$ is the solution to (2)?

In the case of the wave equation, the uniqueness result for the linear inverse problem has been proved by M.V. KLIBANOV in [7] and a stability result of M. YAMAMOTO, deriving from it, can be read in [13]. 
Here we set $y=y(q)$ the weak solution to (1) and $u=u(f)$ the one to (2). If we formally linearize equation (1) around a non stationary solution, we obtain equation (2). In fact, we notice here that if we set $f=q-p, u=y(p)-y(q)$ and $R=y(q)$ on $\Omega \times(0, T)$, we obtain (2) after substraction of (1) with potential $q$ from (1) with potential $p$ and linearization.

In our inverse problem, we have to determine a coefficient of a lower order term in a Schrödinger equation from a single time dependent observation of Neumann data on a part $\Gamma_{0}$ of the boundary. On the other hand, there is another formulation for stationnary inverse problems knowing the Dirichlet to Neumann map and the relation between the two problems is not really clear. In this latter direction, results are given in [2] for the stationnary Schrödinger equation which appear to be similar to ours.

Assuming that $q \in L^{\infty}$ is a given function, we are concerned with the stability around $q$. That is to say $q$ and $y(q)$ are known while $p$ is unknown. Later in section 5, we will give a meaning to equation (1) when $y_{0} \in L^{2}(\Omega)$ and $h \in L^{2}(\partial \Omega \times(0, T))$. Of course, additional assumptions will be required on $y_{0}$ and $h$ in order to obtain our main result which states as follows.

Theorem 1. Let $\mathcal{U}$ be a bounded subset of $L^{\infty}(\Omega), q \in L^{\infty}(\Omega)$ and y be a solution of equation (1).

We assume

$$
\begin{gathered}
\exists x_{0} \in \mathbb{R}^{N} \backslash \bar{\Omega} \text { such that } \Gamma_{0} \supset\left\{x \in \partial \Omega ;\left(x-x_{0}\right) \cdot \nu(x) \geq 0\right\}, \\
\qquad y(q) \in W^{1,2}\left(0, T, L^{\infty}(\Omega)\right), \\
y_{0} \text { is real valued and }\left|y_{0}\right| \geq r_{0}>0 \text {, ae in } \bar{\Omega} .
\end{gathered}
$$

There exists a constant $C=C\left(\Omega, T, \Gamma_{0},\|q\|_{L^{\infty}(\Omega)}, y_{0}, h, \mathcal{U}\right)>0$ such that if

$$
\frac{\partial y(q)}{\partial \nu}-\frac{\partial y(p)}{\partial \nu} \in H^{1}\left(0, T ; L^{2}\left(\Gamma_{0}\right)\right)
$$

then $\forall p \in \mathcal{U}$,

$$
\|q-p\|_{L^{2}(\Omega)} \leq C\left\|\frac{\partial y(q)}{\partial \nu}-\frac{\partial y(p)}{\partial \nu}\right\|_{H^{1}\left(0, T ; L^{2}\left(\Gamma_{0}\right)\right)} .
$$

Remarks : 1) We have the same result if $y_{0}$ takes its values in $i \mathbb{R}$.

2) If we consider equation $(1)$ on $(-T, T)$ and with $y_{0}$ taking its values in $\mathbb{C}$, then, under the formalism of Theorem 1, we can prove the estimate

$$
\|q-p\|_{L^{2}(\Omega)} \leq C\left\|\frac{\partial y(q)}{\partial \nu}-\frac{\partial y(p)}{\partial \nu}\right\|_{H^{1}\left(-T, T ; L^{2}\left(\Gamma_{0}\right)\right)} .
$$

A regularity result in the linear case, obtained in section 4 , implies that the right hand side of inequality (3) is finite under some additional regularity on $y(q)$. 
Corollary 2. Let $\mathcal{U}$ be a bounded subset of $L^{\infty}(\Omega)$ and $q \in L^{\infty}(\Omega)$.

We assume :

$$
\begin{gathered}
\exists x_{0} \in \mathbb{R}^{N} \backslash \bar{\Omega} \text { such that } \Gamma_{0} \supset\left\{x \in \partial \Omega ;\left(x-x_{0}\right) . \nu(x) \geq 0\right\}, \\
y(q) \in W^{1,2}\left(0, T, W^{1, \infty}(\Omega)\right), \\
y_{0} \text { is real valued and }\left|y_{0}\right| \geq r_{0}>0 \text {, ae in } \bar{\Omega} .
\end{gathered}
$$

Then there exists a constant $C=C\left(\Omega, T, \Gamma_{0},\|q\|_{L^{\infty}}, y_{0}, h, \mathcal{U}\right)>0$ such that $\forall p \in \mathcal{U}$ verifying $q-p \in H_{0}^{1}(\Omega)$,

$$
C^{-1}\|q-p\|_{L^{2}(\Omega)} \leq\left\|\frac{\partial y(q)}{\partial \nu}-\frac{\partial y(p)}{\partial \nu}\right\|_{H^{1}\left(0, T ; L^{2}\left(\Gamma_{0}\right)\right)} \leq C\|q-p\|_{H_{0}^{1}(\Omega)} .
$$

The condition on $y(q)$ requires sufficient smoothness on $q, y_{0}$ and $h$ and compatibility conditions for $y_{0}$ and $h$ on $\partial \Omega \times\{0\}$. In particular, $|h(x, 0)| \geq r_{0}>0, x \in \partial \Omega$ must be satisfied since $\left|y_{0}(x)\right| \geq r_{0}>0$, ae in $\bar{\Omega}$ and $h(0)$ has to be real valued.

The first inequality of Corollary 2 in (4) shows the stability of the nonlinear inverse problem and gives uniqueness while the second inequality gives the continuous dependance of the normal derivative of the solution with respect to the potential.

Many of the results we can refer to concern the wave equation. They are related to the same kind of inverse problems of determining a potential, some of them ([10], [13]) with a Dirichlet boundary data and a Neumann measurement and others with a Neumann boundary data and a Dirichlet measurement ([5], [6]). These references are all based upon local or global Carleman estimates. Nevertheless, in our approach, as in [6] for example, in order to prove Theorem 1, we do not use any of the compactnessuniqueness arguments which are required in [13] for the same kind of situation. Indeed, our present proof is based upon a global Carleman estimate (Proposition 3 ) which leads to the result in a direct way.

Up to our knowledge, the result of determination of a time independent potential in Schrödinger equation from a single time dependent measurement on a suitable part of the boundary is new. Let us notice that in the different context of Cauchy problem, V. ISAKOV in [4] uses local Carleman estimates for the Schrödinger equation to prove uniqueness of the solution.

This paper is organized as follows :

We first establish a global Carleman estimate for a Schrödinger equation with a potential (Section 2). This estimate leads us to show a theorem describing uniqueness and stability of the linear inverse problem (Section 3). The idea is inspired by O. Yu. IMANUVILOV and M. YAMAMOTO [6].

Then, after recalling some classical properties of regularity concerning our equations we prove a two sided inequality in the linear case (Section 4).

In section 5, we complete the proof of Theorem 1 and Corollary 2 from the results obtained for the linear problem. In section 6 , under additional hypotheses, we finally 
improve the result of Theorem 1 by showing stability for a stonger norm of $(p-q)$, using there an observability estimate proved from the same Carleman estimate.

\section{A global Carleman estimate}

In this step, we will show a global Carleman estimate concerning a function $v=v(x, t)$ equals to zero on $\partial \Omega \times(-T, T)$ and solution of a Schrödinger equation with a bounded potential.

First, we assume that it is possible to find a regular and positive weight function $\psi=\psi(x)$ defined on $\mathbb{R}^{N}$ and pseudo- convex with respect to the Schrödinger operator. Indeed, we will suppose that $\psi$ verifies the following properties.

- $\psi \in C^{4}\left(\mathbb{R}^{N}\right)$,

- $\psi(x) \geq 0, \forall x \in \Omega$,

- $|\nabla \psi(x)| \geq \beta>0, \forall x \in \Omega$,

- $\exists \Lambda_{1}>0, \exists \varepsilon>0$ such that $\forall \xi \in \mathbb{R}^{N}, \forall \lambda>\Lambda_{1}$, $\lambda|\nabla \psi \cdot \xi|^{2}+D^{2} \psi(\xi, \bar{\xi}) \geq \varepsilon|\xi|^{2}$

- $\nabla \psi \cdot \nu<0, \forall x \in \partial \Omega \backslash \Gamma_{0}$.

A classical answer to the problem of choosing a weight $\psi$ and a geometrical condition upon $\Gamma_{0}$ is the following :

$$
\begin{gathered}
x_{0} \in \mathbb{R}^{N} \backslash \bar{\Omega} \\
\psi=\left|x-x_{0}\right|^{2} \\
\Gamma_{0} \supset\left\{x \in \partial \Omega,\left(x-x_{0}\right) . \nu(x) \geq 0\right\}
\end{gathered}
$$

In this case all the required conditions are satisfied.

Then, for $s>0$ and $\lambda>0$ we define on $\Omega \times(-T, T)$ the functions $\theta$ and $\varphi$ by

$$
\theta(x, t)=\frac{e^{\lambda \psi(x)}}{(T-t)(T+t)} \text { and } \varphi(x, t)=\frac{\alpha-e^{\lambda \psi(x)}}{(T-t)(T+t)}
$$

where $\alpha>\left\|e^{\lambda \psi}\right\|_{L^{\infty}(\Omega)}$. We also set $L v=i v^{\prime}+\Delta v+q v$.

Proposition 3 (Carleman Estimate). Let $q \in L^{\infty}(\Omega),\|q\|_{L^{\infty}} \leq m$ and let $\psi, \theta$ and $\varphi$ satisfy the above conditions. There exists $\Lambda_{0}>0, s_{0}>0$ and a constant $M=M\left(\Omega, T, \Gamma_{0}, \beta, \varepsilon, m, \Lambda_{0}, s_{0}\right)>0$ such that 
for all $\lambda>\Lambda_{0}$ and for all $s>s_{0}$,

$$
\begin{aligned}
& s \lambda \int_{-T}^{T} \int_{\Omega}|\nabla v|^{2} e^{-2 s \varphi} d x d t+s^{3} \lambda^{4} \int_{-T}^{T} \int_{\Omega}|v|^{2} e^{-2 s \varphi} d x d t \\
+ & \int_{-T}^{T} \int_{\Omega}\left(\left|\widetilde{P}_{1} v\right|^{2}+\left|\widetilde{P}_{2} v\right|^{2}\right) e^{-2 s \varphi} d x d t \\
\leq & M \int_{-T}^{T} \int_{\Omega}|L v|^{2} e^{-2 s \varphi} d x d t+M s \lambda \int_{-T}^{T} \int_{\Gamma_{0}} \theta\left|\frac{\partial v}{\partial \nu}\right|^{2} e^{-2 s \varphi} \nabla \psi \cdot \nu d \sigma d t .
\end{aligned}
$$

for all $v$ satisfying

$$
\mid \begin{aligned}
& L v \in L^{2}(\Omega \times(-T, T)), \\
& v \in L^{2}\left(-T, T ; H_{0}^{1}(\Omega)\right), \\
& \frac{\partial v}{\partial \nu} \in L^{2}\left(-T, T ; L^{2}\left(\Gamma_{0}\right)\right),
\end{aligned}
$$

where $\widetilde{P}_{1}$ and $\widetilde{P}_{2}$ will be defined later by (8) and (9).

\section{Proof :}

We can refer to [1] for the general method. The main idea consists in setting $v=e^{s \varphi} w$ and calculating

$$
P w=e^{-s \varphi} L\left(e^{s \varphi} w\right) .
$$

Thus we have

$$
P w=i w^{\prime}+i s \varphi^{\prime} w+\Delta w+2 s \nabla \varphi \cdot \nabla w+s w \Delta \varphi+s^{2}|\nabla \varphi|^{2} w+q w,
$$

and we set

$$
P_{1} w+P_{2} w=P w-q w
$$

where

$$
\begin{aligned}
& P_{1} w=i w^{\prime}+\Delta w+s^{2}|\nabla \varphi|^{2} w \\
& P_{2} w=i s \varphi^{\prime} w+2 s \nabla \varphi \cdot \nabla w+s \Delta \varphi w .
\end{aligned}
$$

We just have represented $P w-q w$ as the sum of adjoint $\left(P_{1}\right)$ and skew-adjoint $\left(P_{2}\right)$ operators. Then,

$$
\begin{aligned}
\int_{-T}^{T} \int_{\Omega}|P w-q w|^{2} d x d t= & \int_{-T}^{T} \int_{\Omega}\left|P_{1} w\right|^{2} d x d t+\int_{-T}^{T} \int_{\Omega}\left|P_{2} w\right|^{2} d x d t \\
& +2 \operatorname{Re} \int_{-T}^{T} \int_{\Omega} P_{1} w \overline{P_{2} w} d x d t
\end{aligned}
$$

where $\bar{z}$ is the conjugate of $z$ and $\operatorname{Re}(z)$ its real part.

As $v \in L^{2}\left(-T, T ; H_{0}^{1}(\Omega)\right)$ and $v^{\prime} \in L^{2}\left(-T, T ; H^{-1}(\Omega)\right)$ (because $L v \in L^{2}(\Omega \times$ $(-T, T))$ ), we have $v \in C\left([-T, T] ; L^{2}(\Omega)\right)$ and $w \in C\left([-T, T] ; L^{2}(\Omega)\right)$ with $w(x, \pm T)=$ 0 . 
We will first look for lower bounds for

$$
R e \int_{-T}^{T} \int_{\Omega} P_{1} w \overline{P_{2} w} d x d t
$$

reminding that

$$
\begin{aligned}
& P_{1} w=i w^{\prime}+\Delta w+s^{2}|\nabla \varphi|^{2} w \\
& \overline{P_{2} w}=-i s \varphi^{\prime} \bar{w}+2 s \nabla \varphi \cdot \nabla \bar{w}+s \Delta \varphi \bar{w} .
\end{aligned}
$$

We multiply each term of $P_{1} w$ by each term of $\overline{P_{2} w}$. The properties of $w$ and some integrations by parts allow to write the following equalities.

$$
I_{11}=\operatorname{Re} \int_{-T}^{T} \int_{\Omega} i w^{\prime}\left(-i s \varphi^{\prime} \bar{w}\right) d x d t=-\frac{s}{2} \int_{-T}^{T} \int_{\Omega} \varphi^{\prime \prime}|w|^{2} d x d t .
$$

Writing $\operatorname{Im}(z)$ for the imaginary part of $z \in \mathbb{C}$, we have $\operatorname{Im}(z)-\operatorname{Im}(\bar{z})=2 \operatorname{Im}(z)$ and taking $z=2 s \lambda \int_{-T}^{T} \int_{\Omega} \theta \nabla \psi \nabla \bar{w} w^{\prime} d x d t$, we show that :

$$
\begin{aligned}
I_{12}= & \operatorname{Re} \int_{-T}^{T} \int_{\Omega} i w^{\prime}(2 s \nabla \varphi \cdot \nabla \bar{w}) d x d t \\
= & s \lambda \operatorname{Im} \int_{-T}^{T} \int_{\Omega} \theta\left(\Delta \psi+\lambda|\nabla \psi|^{2}\right) w \overline{w^{\prime}} d x d t \\
& -s \lambda \operatorname{Im} \int_{-T}^{T} \int_{\Omega} \theta^{\prime} w \nabla \psi \cdot \nabla \bar{w} d x d t .
\end{aligned}
$$

Moreover, since $\operatorname{Im} z=-\operatorname{Im} \bar{z}$, then :

$$
I_{13}=\operatorname{Re} \int_{-T}^{T} \int_{\Omega} i w^{\prime}(s \Delta \varphi \bar{w}) d x d t=-s \lambda \operatorname{Im} \int_{-T}^{T} \int_{\Omega} \theta\left(\Delta \psi+\lambda|\nabla \psi|^{2}\right) w \bar{w}^{\prime} d x d t
$$

and since $\operatorname{Im} \int_{-T}^{T} \int_{\Omega} \varphi^{\prime} \nabla \bar{w} \cdot \nabla w d x d t=0$,

$$
I_{21}=\operatorname{Re} \int_{-T}^{T} \int_{\Omega} \Delta w\left(-i s \varphi^{\prime} \bar{w}\right) d x d t=s \lambda \operatorname{Im} \int_{-T}^{T} \int_{\Omega} \theta^{\prime} \bar{w} \nabla \psi \cdot \nabla w d x d t
$$

The next inequality uses the fact that $\nabla w=\frac{\partial w}{\partial \nu} \cdot \nu$ on $\partial \Omega \times(0, T)$ because $w=0$ on $\partial \Omega \times(0, T):$ 


$$
\begin{aligned}
I_{22}= & \operatorname{Re} \int_{-T}^{T} \int_{\Omega} \Delta w(2 s \nabla \varphi \cdot \nabla \bar{w}) d x d t \\
= & -s \lambda \int_{-T}^{T} \int_{\Omega} \theta\left(\Delta \psi+\lambda|\nabla \psi|^{2}\right)|\nabla w|^{2} d x d t \\
& -s \lambda \int_{-T}^{T} \int_{\partial \Omega} \theta\left|\frac{\partial w}{\partial \nu}\right|^{2} \nabla \psi \cdot \nu d \sigma d t \\
& +2 s \lambda^{2} \int_{-T}^{T} \int_{\Omega} \theta|\nabla \psi \cdot \nabla w|^{2} d x d t \\
& +2 s \lambda \operatorname{Re} \int_{-T}^{T} \int_{\Omega} \theta \sum_{i, j=1}^{n} \frac{\partial \psi}{\partial x_{i} \partial x_{j}} \frac{\partial w}{\partial x_{i}} \frac{\partial \bar{w}}{\partial x_{j}} d x d t .
\end{aligned}
$$

Using integrations by parts we obtain :

$$
\begin{aligned}
I_{23}= & \operatorname{Re} \int_{-T}^{T} \int_{\Omega} \Delta w(s \Delta \varphi \bar{w}) d x d t \\
= & s \lambda \int_{-T}^{T} \int_{\Omega} \theta\left(\Delta \psi+\lambda|\nabla \psi|^{2}\right)|\nabla w|^{2} d x d t \\
& -\frac{s \lambda}{2} \int_{-T}^{T} \int_{\Omega} \theta \Delta^{2} \psi|w|^{2} d x d t \\
& -\frac{s \lambda^{2}}{2} \int_{-T}^{T} \int_{\Omega} \theta\left(|\Delta \psi|^{2}+2 \nabla \psi \cdot \nabla(\Delta \psi)+\Delta\left(|\nabla \psi|^{2}\right)\right)|w|^{2} d x d t \\
& -s \lambda^{3} \int_{-T}^{T} \int_{\Omega} \theta\left(|\nabla \psi|^{2} \Delta \psi+\nabla \psi \cdot \nabla\left(|\nabla \psi|^{2}\right)\right)|w|^{2} d x d t \\
& -\frac{s \lambda^{4}}{2} \int_{-T}^{T} \int_{\Omega} \theta|\nabla \psi|^{4}|w|^{2} d x d t,
\end{aligned}
$$

and we obviously have

$$
\begin{aligned}
I_{31}= & \operatorname{Re} \int_{-T}^{T} \int_{\Omega} s^{2}|\nabla \varphi|^{2} w\left(i s \varphi^{\prime} \bar{w}\right) d x d t=0 \\
I_{32}= & \operatorname{Re} \int_{-T}^{T} \int_{\Omega} s^{2}|\nabla \varphi|^{2} w(2 s \nabla \varphi \cdot \nabla \bar{w}) d x d t \\
= & s^{3} \lambda^{3} \int_{-T}^{T} \int_{\Omega} \theta^{3}\left(|\nabla \psi|^{2} \Delta \psi+\nabla \psi \cdot \nabla\left(|\nabla \psi|^{2}\right)\right)|w|^{2} d x d t \\
& +3 s^{3} \lambda^{4} \int_{-T}^{T} \int_{\Omega} \theta^{3}|\nabla \psi|^{4}|w|^{2} d x d t
\end{aligned}
$$


and

$$
\begin{aligned}
I_{33} & =\operatorname{Re} \int_{-T}^{T} \int_{\Omega} s^{2}|\nabla \varphi|^{2} w(s \Delta \varphi \bar{w}) d x d t \\
& =-s^{3} \lambda^{3} \int_{-T}^{T} \int_{\Omega} \theta^{3}|\nabla \psi|^{2} \Delta \psi|w|^{2} d x d t-s^{3} \lambda^{4} \int_{-T}^{T} \int_{\Omega} \theta^{3}|\nabla \psi|^{4}|w|^{2} d x d t .
\end{aligned}
$$

These four last equalities explain why we required $\psi \in C^{4}\left(\mathbb{R}^{n}\right)$.

Thereafter, we obtain :

$$
\begin{aligned}
& \operatorname{Re} \int_{-T}^{T} \int_{\Omega} P_{1} w \overline{P_{2} w} d x d t= \\
& -\quad \frac{s}{2} \int_{-T}^{T} \int_{\Omega} \varphi^{\prime \prime}|w|^{2} d x d t-2 s \lambda I m \int_{-T}^{T} \int_{\Omega} \theta^{\prime} w \nabla \psi \cdot \nabla \bar{w} d x d t \\
& -\quad s \lambda \int_{-T}^{T} \int_{\partial \Omega} \theta\left|\frac{\partial w}{\partial \nu}\right|^{2} \nabla \psi \cdot \nu d \sigma d t+2 s \lambda^{2} \int_{-T}^{T} \int_{\Omega} \theta|\nabla \psi \cdot \nabla w|^{2} d x d t \\
& +\quad 2 s \lambda \operatorname{Re} \int_{-T}^{T} \int_{\Omega} \theta D^{2} \psi(\nabla w, \nabla \bar{w}) d x d t-\frac{s \lambda}{2} \int_{-T}^{T} \int_{\Omega} \theta \Delta^{2} \psi|w|^{2} d x d t \\
& -\quad \frac{s \lambda^{2}}{2} \int_{-T}^{T} \int_{\Omega} \theta\left(|\Delta \psi|^{2}+2 \nabla \psi \cdot \nabla(\Delta \psi)+\Delta\left(|\nabla \psi|^{2}\right)\right)|w|^{2} d x d t \\
& -\quad s \lambda^{3} \int_{-T}^{T} \int_{\Omega} \theta\left(|\nabla \psi|^{2} \Delta \psi+\nabla \psi \cdot \nabla\left(|\nabla \psi|^{2}\right)\right)|w|^{2} d x d t \\
& -\quad \frac{s \lambda^{4}}{2} \int_{-T}^{T} \int_{\Omega} \theta|\nabla \psi|^{4}|w|^{2} d x d t+s^{3} \lambda^{3} \int_{-T}^{T} \int_{\Omega} \theta^{3} \nabla \psi \cdot \nabla\left(|\nabla \psi|^{2}\right)|w|^{2} d x d t \\
& +\quad 2 s^{3} \lambda^{4} \int_{-T}^{T} \int_{\Omega} \theta^{3}|\nabla \psi|^{4}|w|^{2} d x d t .
\end{aligned}
$$

We call $X_{1}$ the terms which are neglectible within respect to $s \lambda^{2} \int_{-T}^{T} \int_{\Omega} \theta|\nabla \psi \cdot \nabla w|^{2} d x d t$ or $s^{3} \lambda^{4} \int_{-T}^{T} \int_{\Omega} \theta^{3}|\nabla \psi|^{4}|w|^{2} d x d t$.

Then:

$$
\begin{aligned}
X_{1} & =-\frac{s}{2} \int_{-T}^{T} \int_{\Omega} \varphi^{\prime \prime}|w|^{2} d x d t-2 s \lambda I m \int_{-T}^{T} \int_{\Omega} \theta^{\prime} w \nabla \psi \cdot \nabla \bar{w} d x d t \\
& -\frac{s \lambda}{2} \int_{-T}^{T} \int_{\Omega} \theta \Delta^{2} \psi|w|^{2} d x d t \\
& -\frac{s \lambda^{2}}{2} \int_{-T}^{T} \int_{\Omega} \theta\left(|\Delta \psi|^{2}+2 \nabla \psi \cdot \nabla(\Delta \psi)+\Delta\left(|\nabla \psi|^{2}\right)\right)|w|^{2} d x d t \\
& -s \lambda^{3} \int_{-T}^{T} \int_{\Omega} \theta\left(|\nabla \psi|^{2} \Delta \psi+\nabla \psi \cdot \nabla\left(|\nabla \psi|^{2}\right)\right)|w|^{2} d x d t \\
& -\frac{s \lambda^{4}}{2} \int_{-T}^{T} \int_{\Omega} \theta|\nabla \psi|^{4}|w|^{2} d x d t+s^{3} \lambda^{3} \int_{-T}^{T} \int_{\Omega} \theta^{3} \nabla \psi \cdot \nabla\left(|\nabla \psi|^{2}\right)|w|^{2} d x d t .
\end{aligned}
$$


Now, we can notice that :

1) $s \lambda \operatorname{Im} \int_{-T}^{T} \int_{\Omega} \theta^{\prime} w \nabla \psi \cdot \nabla \bar{w} d x d t \leq s \lambda \int_{-T}^{T} \int_{\Omega}\left(\theta^{\prime}\right)^{\frac{1}{2}}|\nabla \psi \cdot \nabla w|^{2} d x d t$

$$
+s \lambda \int_{-T}^{T} \int_{\Omega}\left(\theta^{\prime}\right)^{\frac{3}{2}}|w|^{2} d x d t
$$

2) $\alpha$ is such that $\varphi>0$ on $\Omega \times(-T, T)$,

3) $|\theta| \leq C \theta^{3},\left|\theta^{\prime}\right| \leq C \theta^{2}$ and $\left|\varphi^{\prime \prime}\right| \leq C \theta^{3}$ on $(-T, T) \times \Omega, C=C(T)>0$.

Then,

$$
\begin{aligned}
\left|X_{1}\right| \leq & C s \lambda \int_{-T}^{T} \int_{\Omega} \theta|\nabla \psi \cdot \nabla w|^{2} d x d t+C s \lambda^{4} \int_{-T}^{T} \int_{\Omega} \theta|w|^{2} d x d t \\
& +C s^{3} \lambda^{3} \int_{-T}^{T} \int_{\Omega} \theta^{3}|w|^{2} d x d t .
\end{aligned}
$$

We can also write :

$$
\begin{aligned}
R e \int_{-T}^{T} \int_{\Omega} P_{1} w \overline{P_{2} w} d x d t \geq & X_{1}+2 s \lambda^{2} \int_{-T}^{T} \int_{\Omega} \theta|\nabla \psi \cdot \nabla w|^{2} d x d t \\
& +2 s \lambda \operatorname{Re} \int_{-T}^{T} \int_{\Omega} \theta D^{2} \psi(\nabla w, \nabla \bar{w}) d x d t \\
& +2 s^{3} \lambda^{4} \int_{-T}^{T} \int_{\Omega} \theta^{3}|\nabla \psi|^{4}|w|^{2} d x d t \\
& -s \lambda \int_{-T}^{T} \int_{\partial \Omega} \theta\left|\frac{\partial w}{\partial \nu}\right|^{2} \nabla \psi \cdot \nu d \sigma d t
\end{aligned}
$$

Moreover,

$$
\int_{-T}^{T} \int_{\Omega}|P w-q w|^{2} d x d t \leq 2 \int_{-T}^{T} \int_{\Omega}|P w|^{2} d x d t+2 \int_{-T}^{T} \int_{\Omega} q^{2}|w|^{2} d x d t .
$$

Therefore, from these two last inequalities and if we impose

$$
|\nabla \psi(x)| \geq \beta>0, \quad \forall x \in \Omega,
$$

we obtain :

$$
\begin{aligned}
& \quad 4 s \lambda^{2} \int_{-T}^{T} \int_{\Omega} \theta|\nabla \psi \cdot \nabla w|^{2} d x d t+4 s \lambda \operatorname{Re} \int_{-T}^{T} \int_{\Omega} \theta D^{2} \psi(\nabla w, \nabla \bar{w}) d x d t \\
& +4 s^{3} \lambda^{4} \beta^{4} \int_{-T}^{T} \int_{\Omega} \theta^{3}|w|^{2} d x d t+\int_{-T}^{T} \int_{\Omega}\left|P_{1} w\right|^{2}+\left|P_{2} w\right|^{2} d x d t \\
& \leq \quad 2\left|X_{1}\right|+2 \int_{-T}^{T} \int_{\Omega}|P w|^{2} d x d t+2 \int_{-T}^{T} \int_{\Omega} q^{2}|w|^{2} d x d t \\
& +2 s \lambda \int_{-T}^{T} \int_{\partial \Omega} \theta\left|\frac{\partial w}{\partial \nu}\right|^{2} \nabla \psi \cdot \nu d \sigma d t .
\end{aligned}
$$


Hence, it is clear that if we take $\lambda>\Lambda_{2}$ and $s>s_{0}$ large enough, then $\int_{-T}^{T} \int_{\Omega} q^{2}|w|^{2} d x d t$ and all the terms of $X_{1}$ will be absorbed by the two dominating terms of the left hand side. Then, we see there exists $M_{1}>0$ depending on $\Omega, T, m, \beta, \Lambda_{2}, s_{0}$ and independant of $s$ and $\lambda$ such that

$$
\begin{aligned}
& s \lambda^{2} \int_{-T}^{T} \int_{\Omega} \theta|\nabla \psi \cdot \nabla w|^{2} d x d t+s \lambda R e \int_{-T}^{T} \int_{\Omega} \theta D^{2} \psi(\nabla w, \nabla \bar{w}) d x d t \\
+ & s^{3} \lambda^{4} \int_{-T}^{T} \int_{\Omega} \theta^{3}|w|^{2} d x d t+\int_{-T}^{T} \int_{\Omega}\left(\left|P_{1} w\right|^{2}+\left|P_{2} w\right|^{2}\right) d x d t \\
\leq & M_{1} \int_{-T}^{T} \int_{\Omega}|P w|^{2} d x d t+M_{1} s \lambda \int_{-T}^{T} \int_{\partial \Omega} \theta\left|\frac{\partial w}{\partial \nu}\right|^{2} \nabla \psi \cdot \nu d \sigma d t .
\end{aligned}
$$

At this step, applying condition (5) on $\psi$, we obtain that $\forall \lambda>\Lambda_{0}$, where $\Lambda_{0}=$ $\max \left(\Lambda_{2}, \Lambda_{1}\right)$,

$$
\begin{aligned}
& \varepsilon s \lambda \int_{-T}^{T} \int_{\Omega} \theta|\nabla w|^{2} d x d t+s^{3} \lambda^{4} \beta^{4} \int_{-T}^{T} \int_{\Omega} \theta^{3}|w|^{2} d x d t \\
+ & \int_{-T}^{T} \int_{\Omega}\left|P_{1} w\right|^{2} d x d t+\int_{-T}^{T} \int_{\Omega}\left|P_{2} w\right|^{2} d x d t \\
\leq & M_{1} \int_{-T}^{T} \int_{\Omega}|P w|^{2} d x d t+M_{1} s \lambda \int_{-T}^{T} \int_{\partial \Omega} \theta\left|\frac{\partial w}{\partial \nu}\right|^{2} \nabla \psi \cdot \nu d \sigma d t .
\end{aligned}
$$

Let us remark that $\theta>0$ on $(-T, T) \times \Omega$ and $\nabla \psi \cdot \nu<0$ on $\partial \Omega \backslash \Gamma_{0}$. Then, by modifying the constant $M_{1}$ into $M_{2}=M_{2}\left(\Omega, T, m, \beta, \varepsilon, \Lambda_{0}, s_{0}\right)>0$ we obtain :

$$
\begin{aligned}
& s \lambda \int_{-T}^{T} \int_{\Omega}|\nabla w|^{2} d x d t+s^{3} \lambda^{4} \int_{-T}^{T} \int_{\Omega}|w|^{2} d x d t \\
& +\int_{-T}^{T} \int_{\Omega}\left(\left|P_{1} w\right|^{2}+\left|P_{2} w\right|^{2}\right) d x d t \\
& \leq M_{2} \int_{-T}^{T} \int_{\Omega}|P w|^{2} d x d t+M_{2} s \lambda \int_{-T}^{T} \int_{\Gamma_{0}} \theta\left|\frac{\partial w}{\partial \nu}\right|^{2} \nabla \psi \cdot \nu d \sigma d t .
\end{aligned}
$$

We can now rewrite our inequality with $v$ instead of $w$. We have

$$
\begin{aligned}
& |v|^{2} e^{-2 s \varphi}=|w|^{2}, \\
& e^{-2 s \varphi}|\nabla v|^{2}=|\nabla w+s \nabla \varphi w|^{2} \leq 2|\nabla w|^{2}+2 s^{2}|\nabla \varphi|^{2}|w|^{2}, \\
& \left|\frac{\partial v}{\partial \nu}\right|^{2} e^{-2 s \varphi}=\left|\frac{\partial w}{\partial \nu}\right|^{2} \text { on } \partial \Omega, \\
& P w=e^{-s \varphi} L(v),
\end{aligned}
$$

and defining

$$
\begin{aligned}
& \widetilde{P}_{1} v=e^{s \varphi} P_{1} w, \\
& \widetilde{P}_{2} v=e^{s \varphi} P_{2} w,
\end{aligned}
$$


we finally show (7): $\exists M=M\left(\Omega, T, \Gamma_{0}, \beta, \varepsilon, m, \Lambda_{0}, s_{0}\right)$ such that $\forall s>s_{0}, \forall \lambda>$ $\Lambda_{0}$

$$
\begin{aligned}
& s \lambda \int_{-T}^{T} \int_{\Omega}|\nabla v|^{2} e^{-2 s \varphi} d x d t+s^{3} \lambda^{4} \int_{-T}^{T} \int_{\Omega}|v|^{2} e^{-2 s \varphi} d x d t \\
+ & \int_{-T}^{T} \int_{\Omega}\left(\left|\widetilde{P}_{1} v\right|^{2}+\left|\widetilde{P}_{2} v\right|^{2}\right) e^{-2 s \varphi} d x d t \\
\leq & M \int_{-T}^{T} \int_{\Omega}|L v|^{2} e^{-2 s \varphi} d x d t+M s \lambda \int_{-T}^{T} \int_{\Gamma_{0}} \theta\left|\frac{\partial v}{\partial \nu}\right|^{2} e^{-2 s \varphi} \nabla \psi \cdot \nu d \sigma d t .
\end{aligned}
$$

Hence the end of the proof of Proposition 3.

Remark : Under the conditions upon $\psi$, we can notice that $\theta e^{-2 s \varphi}$ and $\nabla \psi \cdot \nu$ are bounded on $(-T, T) \times \Gamma_{0}$ and replace

$$
\int_{-T}^{T} \int_{\Gamma_{0}} \theta\left|\frac{\partial v}{\partial \nu}\right|^{2} e^{-2 s \varphi} \nabla \psi \cdot \nu d \sigma d t \text { by } C \int_{-T}^{T} \int_{\Gamma_{0}}\left|\frac{\partial v}{\partial \nu}\right|^{2} d \sigma d t
$$

We knew, from counter-examples that a geometrical condition was necessary and the choice of $\Gamma_{0}$ given in (6) is very usual. Indeed, E. MACHTYNGIER [9], R. TRIGGIANI, P.- F. YAO and I. LASIECKA in references [8], [11] and [12] used that kind of open set of the boundary. In [9], E. MACHTYNGIER shows an observability inequality which estimates initial data by boundary Neumann data for a Schrödinger equation without a potential on $\Gamma_{0}=\left\{x \in \partial \Omega ;\left(x-x_{0}\right) \cdot \nu(x) \geq 0\right\}$ using a multiplier identity and Holmgren's uniqueness theorem. Moreover, observability inequalities are technically related to our inverse problem (see [13] and section 6). The reference [11] is based on Carleman estimates, that R. TRIGGIANI proved for a more general kind of coupled Schrödinger equation and applied it to exact controllability. However, both of them are not directly applicable to obtain the result we are expecting.

\section{Stability in the linear case}

We first consider the linear inverse problem and give the following result.

Theorem 4. Let $q \in L^{\infty}(\Omega)$ and $u$ be a solution of equation (2).

We assume that

$$
\begin{gathered}
\Gamma_{0} \text { satisfies }(6), \\
R \in W^{1,2}\left(0, T, L^{\infty}(\Omega)\right), \\
R(0) \text { is real valued and }|R(x, 0)| \geq r_{0}>0, \text { ae in } \bar{\Omega} \text {. }
\end{gathered}
$$

There exists a constant $C=C\left(\Omega, T,\|q\|_{L^{\infty}(\Omega)}, R\right)>0$ such that if

$$
\frac{\partial u}{\partial \nu} \in H^{1}\left(0, T ; L^{2}\left(\Gamma_{0}\right)\right)
$$


then,

$$
\|f\|_{L^{2}(\Omega)} \leq C\left\|\frac{\partial u}{\partial \nu}\right\|_{H^{1}\left(0, T ; L^{2}\left(\Gamma_{0}\right)\right)} .
$$

Proof :

As we need to estimate $\frac{\partial u}{\partial \nu}$ in $H^{1}\left(0, T ; L^{2}\left(\Gamma_{0}\right)\right)$ norm, we work on the equation satisfied by $v=u^{\prime}$ :

$$
\left\{\begin{array}{l}
i v^{\prime}(x, t)+\Delta v(x, t)+q(x) v(x, t)=f(x) R^{\prime}(x, t), x \in \Omega, t \in(0, T) \\
v(x, t)=0, x \in \partial \Omega, t \in(0, T) \\
v(x, 0)=-i f(x) R(x, 0), x \in \Omega
\end{array}\right.
$$

The Carleman inequality we just obtained is the key of the proof. We extend the function $v$ on $\Omega \times(-T, T)$ by the formula $v(x, t)=-\bar{v}(x,-t)$ for every $(x, t) \in$ $\Omega \times(-T, 0)$. Since $R(0)$ and $f$ are real valued, $v \in C\left([-T, T] ; H_{0}^{1}(\Omega)\right)$ and $\frac{\partial v}{\partial \nu} \in$ $L^{2}((-T, T) \times \Gamma)$. We also extend $R$ on $\Omega \times(-T, T)$ by the formula $R(x, t)=$ $\bar{R}(x,-t)$ for every $(x, t) \in \Omega \times(-T, 0)$ and if we denote the extention of $R^{\prime}$ by the same notation, then $R^{\prime} \in L^{2}\left(-T, T ; W^{1, \infty}(\Omega)\right)$. Thus, $v$ satisfies the same equation (11), set in $(-T, T)$.

We set $w=e^{-s \varphi} v, P_{1} w=i w^{\prime}+\Delta w+s^{2}|\nabla \varphi|^{2} w$ and $e^{-s \varphi} \widetilde{P}_{1} v=P_{1} w$ as in section 2 and we define :

$$
I=I m \int_{-T}^{0} \int_{\Omega} \widetilde{P}_{1} v e^{-2 s \varphi} \bar{v} d x d t .
$$

On the one hand,

$$
\begin{aligned}
I & =\operatorname{Im} \int_{-T}^{0} \int_{\Omega} P_{1} w \bar{w} d x d t \\
& =\operatorname{Im} \int_{-T}^{0} \int_{\Omega}\left(i w^{\prime}+\Delta w+s^{2}|\nabla \varphi|^{2} w\right) \bar{w} d x d t \\
& =\operatorname{Re} \int_{-T}^{0} \int_{\Omega} w^{\prime} \bar{w} d x d t-I m \int_{-T}^{0} \int_{\Omega}\left(|\nabla w|^{2}-s^{2}|\nabla \varphi|^{2}|w|^{2}\right) d x d t \\
& =\frac{1}{2} \int_{-T}^{0} \int_{\Omega}\left(|w|^{2}\right)^{\prime} d x d t \\
& =\frac{1}{2} \int_{\Omega}|w(x, 0)|^{2} d x \\
& =\frac{1}{2} \int_{\Omega}|f(x)|^{2}|R(x, 0)|^{2} e^{-2 s \varphi(x, 0)} d x
\end{aligned}
$$

On the other hand, Cauchy-Schwarz inequality and Carleman estimate stated as in 
Proposition 3 in section 2, give :

$$
\begin{aligned}
I & \leq\left(\int_{-T}^{T} \int_{\Omega}\left|\widetilde{P}_{1} v\right|^{2} e^{-2 s \varphi} d x d t\right)^{\frac{1}{2}}\left(\int_{-T}^{T} \int_{\Omega}|v|^{2} e^{-2 s \varphi} d x d t\right)^{\frac{1}{2}} \\
& \leq s^{-\frac{3}{2}}\left(M \int_{-T}^{T} \int_{\Omega}\left|f R^{\prime}\right|^{2} e^{-2 s \varphi} d x d t+M s \int_{-T}^{T} \int_{\Gamma_{0}} \theta\left|\frac{\partial v}{\partial \nu}\right|^{2} e^{-2 s \varphi} \nabla \psi \cdot \nu d \sigma d t\right)
\end{aligned}
$$

Then, $\varphi(x, t)=\frac{\alpha-e^{\lambda \psi(x)}}{(T-t)(T+t)}$ is such that $e^{-2 s \varphi(x, t)} \leq e^{-2 s \varphi(x, 0)}$ for all $x \in \Omega$ and $t \in(-T, T)$ and it is easy to see that under the conditions satisfied by $\psi, \theta e^{-2 s \varphi}$ and $\nabla \psi . \nu$ are bounded on $(-T, T) \times \Gamma_{0}$. Therefore

$$
I \leq s^{-\frac{3}{2}}\left(M \int_{-T}^{T} \int_{\Omega}\left|f R^{\prime}\right|^{2} e^{-2 s \varphi(x, 0)} d x d t+M s \int_{-T}^{T} \int_{\Gamma_{0}}\left|\frac{\partial v}{\partial \nu}\right|^{2} d \sigma d t\right) .
$$

Moreover, using the definition of the extensions of $v$ and $R^{\prime}$, we easily get

$$
I \leq s^{-\frac{3}{2}}\left(M \int_{0}^{T} \int_{\Omega}\left|f R^{\prime}\right|^{2} e^{-2 s \varphi(x, 0)} d x d t+M s \int_{0}^{T} \int_{\Gamma_{0}}\left|\frac{\partial v}{\partial \nu}\right|^{2} d \sigma d t\right) .
$$

From $R \in W^{1,2}\left(0, T, L^{\infty}(\Omega)\right)$ and $|R(x, 0)| \geq r_{0}>0$ ae in $\bar{\Omega}$, we deduce that :

$$
\exists g_{0} \in L^{2}(0, T),\left|R^{\prime}(x, t)\right| \leq g_{0}(t)|R(x, 0)|, \forall x \in \Omega, t \in(0, T) .
$$

Hence we have :

$$
\begin{aligned}
\int_{\Omega}|f|^{2}|R(0)|^{2} e^{-2 s \varphi(0)} d x & \leq M s^{-\frac{3}{2}} \int_{0}^{T} \int_{\Omega}|f|^{2}\left|g_{0}\right|^{2}|R(0)|^{2} e^{-2 s \varphi(0)} d x d t \\
& +M s^{-\frac{1}{2}} \int_{0}^{T} \int_{\Gamma_{0}}\left|\frac{\partial v}{\partial \nu}\right|^{2} d \sigma d t .
\end{aligned}
$$

But $g_{0} \in L^{2}(0, T) \Rightarrow \int_{0}^{T}\left|g_{0}(t)\right|^{2} d t \leq K<+\infty$ and so we write

$$
\left[\int_{\Omega}|f(x)|^{2}|R(x, 0)|^{2} e^{-2 s \varphi(x, 0)} d x\right]\left(1-\frac{M K}{s^{\frac{3}{2}}}\right) \leq M s^{-\frac{1}{2}} \int_{0}^{T} \int_{\Gamma_{0}}\left|\frac{\partial v}{\partial \nu}\right|^{2} d \sigma d t .
$$

Then, if $s$ is large enough, $\left(s>(M K)^{\frac{2}{3}}\right)$, we see that there exist a constant $C=$ $C(M, s)>0$ such that :

$$
\int_{\Omega}|f(x)|^{2}|R(x, 0)|^{2} e^{-2 s \varphi(x, 0)} d x \leq C \int_{0}^{T} \int_{\Gamma_{0}}\left|\frac{\partial v}{\partial \nu}\right|^{2} d \sigma d t .
$$

Since $|R(x, 0)| \geq r_{0}>0$, ae in $\bar{\Omega}$ and $e^{-2 s \varphi(x, 0)} \geq e^{-2 s \frac{\alpha-1}{T^{2}}}>0, \forall x \in \Omega$, we obtain

$$
\int_{\Omega}|f(x)|^{2} d x \leq C \int_{0}^{T} \int_{\Gamma_{0}}\left|\frac{\partial v}{\partial \nu}\right|^{2} d \sigma d t
$$


and it is (10):

$$
\|f\|_{L^{2}(\Omega)} \leq C\left\|\frac{\partial u}{\partial \nu}\right\|_{H^{1}\left(0, T ; L^{2}\left(\Gamma_{0}\right)\right)} .
$$

Therefore, Theorem 4 has been proved.

Remark : if we replace the assumption " $R(0)$ is real valued" by the following " $R(0)$ takes its values in $i \mathbb{R}$ ", using the same idea, but with a different extension of $v$ and $R$, we will be able to prove the same result.

Corollary 5. Let $u$ be the solution of equation (2) and $\Gamma_{0}$ given by (6).

We assume :

$$
q \in L^{\infty}(\Omega), R \in W^{1,2}\left(0, T, W^{1, \infty}(\Omega)\right),
$$

$$
R(0) \text { is real valued and }|R(x, 0)| \geq r_{0}>0 \text { ae in } \bar{\Omega} \text {. }
$$

Then, there exists a constant $C=C\left(\Omega, T,\|q\|_{L^{\infty}(\Omega)}, R\right)>0$ such that for all $f \in$ $H_{0}^{1}(\Omega)$ :

$$
C^{-1}\|f\|_{L^{2}(\Omega)} \leq\left\|\frac{\partial u}{\partial \nu}\right\|_{H^{1}\left(0, T ; L^{2}\left(\Gamma_{0}\right)\right)} \leq C\|f\|_{H_{0}^{1}(\Omega)} .
$$

The proof will be given in the following section.

Remark : It follows from Theorem 4 that

$$
\left(\frac{\partial u}{\partial \nu}=0 \text { on }(0, T) \times \Gamma_{0}\right) \Rightarrow(f=0 \text { on } \Omega)
$$

and it corresponds to the uniqueness result, for the linear case, proposed in section 1. In the non linear situation, we easily show uniqueness by choosing $f=q-p$, $u=y(p)-y(q)$ and $R=y(p)$ on $\Omega \times(0, T)$.

This result can be writen in the following way :

Theorem 6. Let $p \in L^{\infty}(\Omega)$ and $q \in L^{\infty}(\Omega)$. We assume that $y(p)$ or $y(q)$ belongs to $W^{1,2}\left(0, T, W^{1, \infty}(\Omega)\right)$, $y_{0}$ is real valued and $\left|y_{0}(x)\right| \geq r_{0}>0$ almost everywhere in $\bar{\Omega}$.

$$
\text { If } \frac{\partial y(q)}{\partial \nu}=\frac{\partial y(p)}{\partial \nu} \text { on }(0, T) \times \Gamma_{0}, \text { then } q=p \text { on } \Omega \text {. }
$$

\section{Existence and regularity properties}

The estimates we will need to prove Corollary 5 can be summed up by the following lemmas.

Lemma 7. Let us consider

$$
\left\{\begin{array}{l}
i y^{\prime}(x, t)+\Delta y(x, t)+q(x) y(x, t)=g(x, t), x \in \Omega, t \in(0, T) \\
y(x, t)=0, x \in \partial \Omega, t \in(0, T) \\
y(x, 0)=y_{0}(x), x \in \Omega
\end{array}\right.
$$


where $q \in L^{\infty}(\Omega)$, $y_{0} \in H_{0}^{1}(\Omega)$ and $g \in X$, with $X=L^{1}\left(0, T, H_{0}^{1}(\Omega)\right)$ or $X=$ $W^{1,1}\left(0, T, L^{2}(\Omega)\right)$. This equation admits a unique weak solution

$y \in C\left([0, T], H_{0}^{1}(\Omega)\right)$ such that the mapping $\left(g, y_{0}\right) \rightarrow \frac{\partial y}{\partial \nu}$ is linear and continuous from $X \times H_{0}^{1}(\Omega)$ to $L^{2}(\Gamma \times(0, T))$ and $\exists C=C\left(\Omega, T,\|q\|_{L^{\infty}(\Omega)}\right)>0$ such that :

$$
\begin{aligned}
& \forall t \in(0, T), \quad\|y(t)\|_{H_{0}^{1}(\Omega)} \leq C\left(\left\|y_{0}\right\|_{H_{0}^{1}(\Omega)}+\|g\|_{X}\right) \\
& \left\|\frac{\partial y}{\partial \nu}\right\|_{L^{2}(\Gamma \times(0, T))} \leq C\left(\left\|y_{0}\right\|_{H_{0}^{1}(\Omega)}+\|g\|_{X}\right)
\end{aligned}
$$

\section{Proof :}

Concerning estimate (14), we can refer to [3]. It is a classical result which can be formally obtained by two manipulations using Gronwall inequality. In a first time we have to work on " $\operatorname{Im} \int_{\Omega}(13) . \bar{y} d x$ " and show that if $y_{0} \in L^{2}(\Omega)$ and $g \in L^{1}\left(0, T, L^{2}(\Omega)\right)$ then (13) admits a unique solution $y \in C\left([0, T], L^{2}(\Omega)\right)$ such that $\forall t \in(0, T)$,

$$
\|y(t)\|_{L^{2}(\Omega)} \leq C\left(\left\|y_{0}\right\|_{L^{2}(\Omega)}+\|g\|_{L^{1}\left(0, T, L^{2}(\Omega)\right)}\right) .
$$

Then, in a second time, working on " $R e \int_{\Omega}(13) \cdot \overline{y^{\prime}} d x$ ", we manage to obtain (14) in both of the two cases for space $X$.

Estimate (15) can be deduced from this other result :

Lemma 8. Let $\gamma=\gamma(x, t) \in C^{2}\left(\overline{\Omega \times(0, T)}, \mathbb{R}^{n}\right)$. Under the same hypothesis as in the preceding lemma, the following multipliers identity holds for every weak solution of (13) with initial data $y_{0} \in H_{0}^{1}(\Omega)$ and $g \in X$ :

$$
\begin{aligned}
& \int_{0}^{T} \int_{\Gamma} \gamma \cdot \nu\left|\frac{\partial y}{\partial \nu}\right|^{2} d \sigma d t=\left.\operatorname{Im} \int_{\Omega} y \gamma \cdot \nabla \bar{y} d x\right|_{0} ^{T} \\
& +\operatorname{Re} \int_{0}^{T} \int_{\Omega} y \nabla(\operatorname{div} \gamma) \cdot \nabla \bar{y} d x d t+2 \operatorname{Re} \int_{0}^{T} \int_{\Omega} \sum_{i, j=1}^{n} \frac{\partial \gamma_{j}}{\partial x_{i}} \frac{\partial y}{\partial x_{i}} \frac{\partial \bar{y}}{\partial x_{j}} d x d t \\
& -2 \operatorname{Re} \int_{0}^{T} \int_{\Omega} q y \gamma \cdot \nabla \bar{y} d x d t-\operatorname{Re} \int_{0}^{T} \int_{\Omega} q|y|^{2} \operatorname{div} \gamma d x d t \\
& +2 \operatorname{Re} \int_{0}^{T} \int_{\Omega} g \gamma \cdot \nabla \bar{y} d x d t+\operatorname{Re} \int_{0}^{T} \int_{\Omega} g \bar{y} \operatorname{div} \gamma d x d t .
\end{aligned}
$$

We first obtain this identity for very regular data $g \in W^{1,1}(0, T, \mathcal{D}(\Omega))$ and $y_{0} \in$ $\mathcal{D}(\Omega)$ by calculating

$$
\text { " } R e \int_{0}^{T} \int_{\Omega}(13) \cdot\left(\gamma \cdot \nabla \bar{y}+\frac{1}{2} \bar{y} \operatorname{div} \gamma\right) d x d t " .
$$

and the result holds by integration by parts. At this step, reference [9] gives a similar result for the Schrödinger equation without potential. Then, by density, the estimate 
holds true for every solution of (13) with initial data $y_{0} \in H_{0}^{1}(\Omega)$ and $g \in X$.

We finally choose $\gamma=\gamma(x) \in C^{2}\left(\overline{\Omega \times(0, T)}, \mathbb{R}^{n}\right)$ such that $\gamma=\nu$ on the $C^{2}$ boundary $\partial \Omega$. Then $\gamma \cdot \nu=1$ on $(0, T) \times \partial \Omega$ and applying estimate (14) with Lemma 8 , we manage to obtain estimate (15).

\section{Proof of Corollary 5 :}

Since $f \in H_{0}^{1}(\Omega)$ and $R \in W^{1,2}\left(0, T, W^{1, \infty}(\Omega)\right)$, we have $f R^{\prime} \in L^{1}\left(0, T, H_{0}^{1}(\Omega)\right)$ and $f R(0) \in H_{0}^{1}(\Omega)$. Thereafter, we know that equation (11) has a solution $v \in$ $C\left([0, T] ; H_{0}^{1}(\Omega)\right)$ and it also implies $\frac{\partial v}{\partial \nu} \in L^{2}((0, T) \times \Gamma)$. Of course, the left inequality in (12) derives from Theorem 4 . Besides, from Lemma 7 :

$$
\begin{aligned}
\left\|\frac{\partial v}{\partial \nu}\right\|_{L^{2}\left((0, T) \times \Gamma_{0}\right)} & \leq\left\|\frac{\partial v}{\partial \nu}\right\|_{L^{2}((0, T) \times \partial \Omega)} \\
& \leq C\left(\|f R(0)\|_{H_{0}^{1}(\Omega)}+\left\|f R^{\prime}\right\|_{L^{1}\left(0, T, H_{0}^{1}(\Omega)\right)}\right) \\
& \leq C\|f\|_{H_{0}^{1}(\Omega)}
\end{aligned}
$$

This proves the right hand side of inequality (12) and the proof of Corollary 5 is complete.

\section{Proof of Theorem 1 and Corollary 2}

We would like first to give a meaning to equation (1) we are studying.

Lemma 9. Let $q \in L^{\infty}(\Omega), y_{0} \in L^{2}(\Omega)$ and $h \in L^{2}(\partial \Omega \times(0, T))$. Then, there exists a unique solution

$$
y \in C\left([0, T], H^{-1}(\Omega)\right) \cap H^{-1}\left(0, T, L^{2}(\Omega)\right),
$$

defined by transposition, of the problem (1) :

$$
\left\{\begin{array}{l}
i y^{\prime}(x, t)+\Delta y(x, t)+q(x) y(x, t)=0, x \in \Omega, t \in(0, T) \\
y(x, t)=h(x, t), x \in \partial \Omega, t \in(0, T) \\
y(x, 0)=y_{0}(x), x \in \Omega
\end{array}\right.
$$

\section{Proof :}

We define the adjoint system

$$
\begin{cases}i \varphi^{\prime}+\Delta \varphi+q \varphi=g, & \text { in } \Omega \times(0, T) \\ \varphi=0, & \text { on } \partial \Omega \times(0, T) \\ \varphi(T)=0, & \text { in } \Omega\end{cases}
$$

It is well-known that we have the following regularity properties about this Schrödinger equation : 
a) If $g \in L^{1}\left(0, T, H_{0}^{1}(\Omega)\right)$ then $\varphi \in C\left([0, T], H_{0}^{1}(\Omega)\right)$ and $\frac{\partial \varphi}{\partial \nu} \in L^{2}(\Gamma \times(0, T))$ (Lemma 7).

b) If $g \in H_{0}^{1}\left(0, T, L^{2}(\Omega)\right) \hookrightarrow W^{1,1}\left(0, T, L^{2}(\Omega)\right)$ then $\varphi \in C\left([0, T], H^{2}(\Omega)\right)$ and $\frac{\partial \varphi}{\partial \nu} \in C\left([0, T], H^{\frac{1}{2}}(\partial \Omega)\right)$.

Indeed, we first have $\varphi \in C\left([0, T], L^{2}(\Omega)\right)$. The study of the equation satisfied by $\varphi^{\prime}$ gives $\varphi \in C^{1}\left([0, T], L^{2}(\Omega)\right)$ and that leads to $\Delta \varphi=g-q \varphi-i \varphi^{\prime} \in C\left([0, T], L^{2}(\Omega)\right)$. Then $\varphi \in C\left([0, T], H^{2}(\Omega)\right)$ and its normal derivative is in $C\left([0, T], H^{\frac{1}{2}}(\partial \Omega)\right)$.

We say that $y$ is a solution of (1) in the transposition sense if and only if it is possible, for every $g$, to give a meaning to

$$
\int_{0}^{T} \int_{\Omega} \bar{g} y d x d t=i \int_{\Omega} y_{0} \bar{\varphi}(0) d x+\int_{0}^{T} \int_{\partial \Omega} h \frac{\partial \bar{\varphi}}{\partial \nu} d \sigma d t .
$$

We take $g \in \mathcal{D}(0, T, \mathcal{D}(\Omega))$. By density in $L^{1}\left(0, T, H_{0}^{1}(\Omega)\right)$ and $H_{0}^{1}\left(0, T, L^{2}(\Omega)\right)$ we are able to define $y \in L^{\infty}\left(0, T, H^{-1}(\Omega)\right) \cap H^{-1}\left(0, T, L^{2}(\Omega)\right)$ with $q \in L^{\infty}(\Omega)$, $y_{0} \in L^{2}(\Omega)$ and $h \in L^{2}(\partial \Omega \times(0, T))$.

We refer to [9] for the transposition method concerning the Schrödinger equation and the way to prove that we finally obtain $y \in C\left([0, T], H^{-1}(\Omega)\right) \cap H^{-1}\left(0, T, L^{2}(\Omega)\right)$. Nevertheless, we would like to underline that the important point here is that we have a potential $q \in L^{\infty}(\Omega)$ and we have to give a meaning to $q y$ (indeed, we proved that $q y \in H^{-1}\left(0, T ; L^{2}(\Omega)\right)$, since $\left.y \in H^{-1}\left(0, T ; L^{2}(\Omega)\right)\right)$. Let us also notice that the regularity we obtain in $y$ implies $\frac{\partial y}{\partial \nu} \in H^{-2}\left(0, T ; H^{-\frac{3}{2}}(\Gamma)\right)$.

Thereafter, we define $u=y(p)-y(q)$, which verifies :

$$
\left\{\begin{array}{l}
i u^{\prime}+\Delta u+p u=(q-p) y(q), \quad \text { in } \Omega \times(0, T) \\
u=0, \text { on } \partial \Omega \times(0, T) \\
u(0)=0, \text { in } \Omega
\end{array}\right.
$$

The key of our proof is that in the linear case, all the constants depend on the $L^{\infty}$ norm of the potential. Then, since $p \in \mathcal{U}$, where $\mathcal{U}$ is bounded in $L^{\infty}$, we are in fact, with (16) in a situation similar to the linear case (2).

\section{Proof of Theorem 1 :}

We have $y(q) \in W^{1,2}\left(0, T, L^{\infty}(\Omega)\right)$ and we know that

$$
W^{1,2}\left(0, T, L^{\infty}(\Omega)\right) \subset C\left([0, T], W^{1, \infty}(\Omega)\right)
$$

then we have $y(x, 0)=y_{0} \in L^{\infty}(\Omega)$. Thus, hypothesis $\left|y_{0}(x)\right| \geq r_{0}>0$, ae in $\bar{\Omega}$ makes sense and we can apply the result of Theorem 4 , which leads to :

$$
\|q-p\|_{L^{2}(\Omega)} \leq C\left\|\frac{\partial u}{\partial \nu}\right\|_{H^{1}\left(0, T ; L^{2}\left(\Gamma_{0}\right)\right)} .
$$


And the proof of Theorem 1 is complete.

\section{Proof of Corollary 2 :}

We assume that $y(q) \in W^{1,2}\left(0, T, W^{1, \infty}(\Omega)\right)$ and $q-p \in H_{0}^{1}(\Omega)$. Then, it comes $(q-$ p) $y(q) \in W^{1,2}\left(0, T, H_{0}^{1}(\Omega)\right)$ and there exists a unique solution $u \in C\left([0, T], H_{0}^{1}(\Omega)\right)$ to $(16)$.

We are in the same situation as in the linear case (2) and we can apply the result of Corollary 5 which leads to :

$$
C^{-1}\|q-p\|_{L^{2}(\Omega)} \leq\left\|\frac{\partial u}{\partial \nu}\right\|_{H^{1}\left(0, T ; L^{2}\left(\Gamma_{0}\right)\right)} \leq C\|q-p\|_{H_{0}^{1}(\Omega)} .
$$

It means that there exists a constant $C=C\left(\Omega, T, \Gamma_{0},\|q\|_{L^{\infty}}, y_{0}, h, \mathcal{U}\right)>0$ such that for all $p \in \mathcal{U}$ satisfying $q-p \in H_{0}^{1}(\Omega)$,

$$
C^{-1}\|q-p\|_{L^{2}(\Omega)} \leq\left\|\frac{\partial y(q)}{\partial \nu}-\frac{\partial y(p)}{\partial \nu}\right\|_{H^{1}\left(0, T ; L^{2}\left(\Gamma_{0}\right)\right)} \leq C\|q-p\|_{H_{0}^{1}(\Omega)},
$$

and the proof is complete.

Remark : The importance of Carleman estimate being global has to be underlined. It is also well shown, in reference [6], how a global Carleman estimate leads really faster to a conclusion in a non linear situation for the wave equation. We can refer to [13] for a situation using only a local estimate with a weaker result. Indeed, to prove a stability inequality in a non linear situation from the knowledge of the linear case, an observability inequality and a compactness-uniqueness argument are required. However, to improve our results, we will precisely use an observability estimate.

\section{Improvement of the symetry of the two-sided esti- mates}

It is known that for the wave equation, a symetric two sided estimate can be shown, for instance in [13]. The result obtained for the Schrödinger equation in Corollary 2 is not symetric in terms of the norms of $(p-q)$. We will here improve the result of Theorem 1 under slightly stronger regularity hypothesis on $y(q)$.

Proposition 10 (Observability Estimate). We assume $q \in L^{\infty}, z_{0} \in H_{0}^{1}(\Omega)$ and $\Gamma_{0}$ is given by (6). If $z$ is the weak solution of

$$
\left\{\begin{array}{l}
i z^{\prime}(x, t)+\Delta z(x, t)+q(x) z(x, t)=0, x \in \Omega, t \in(0, T) \\
z(x, t)=0, x \in \partial \Omega, t \in(0, T) \\
z(x, 0)=z_{0}(x), x \in \Omega
\end{array}\right.
$$

then, there exists a constant $C=C\left(\Omega, T, \Gamma_{0},\|q\|_{L^{\infty}}\right)>0$ such that

$$
\left\|z_{0}\right\|_{H_{0}^{1}(\Omega)} \leq C\left\|\frac{\partial z}{\partial \nu}\right\|_{L^{2}\left(0, T ; L^{2}\left(\Gamma_{0}\right)\right)} .
$$




\section{Proof :}

It is a well-known consequence of the Carleman estimate (Proposition 3).

Let $0<T_{0}<T_{1}<T$. First of all, since all the needed conditions are satisfied (from Lemma 7), we can apply Proposition 3 to the weak solution $z$ of (17).

Remark : Some changes are made. We work on $[0, T]$ and with

$$
\theta(x, t)=\frac{e^{\lambda \psi(x)}}{T(T-t)}, \varphi(x, t)=\frac{\alpha-e^{\lambda \psi(x)}}{T(T-t)} .
$$

Then we have :

$$
\int_{0}^{T} \int_{\Omega}|\nabla z|^{2} e^{-2 s \varphi} d x d t+s^{2} \lambda^{3} \int_{0}^{T} \int_{\Omega}|z|^{2} e^{-2 s \varphi} d x d t \leq M \int_{0}^{T} \int_{\Gamma_{0}}\left|\frac{\partial z}{\partial \nu}\right|^{2} d \sigma d t
$$

and since $e^{-2 s \varphi} \geq c>0$ on $\left[T_{0}, T_{1}\right] \times \Omega$, it means that :

$$
\|z\|_{L^{2}\left(T_{0}, T_{1} ; H_{0}^{1}(\Omega)\right)} \leq C\left\|\frac{\partial z}{\partial \nu}\right\|_{L^{2}\left((0, T) \times \Gamma_{0}\right)}
$$

Let $\chi \in C^{\infty}(0, T)$ be a function such that $0 \leq \chi(t) \leq 1, \forall t \in[0, T], \chi=1$ on $\left[0, T_{0}\right]$ and $\chi=0$ on $\left[T_{1}, T\right]$. Then, $\chi^{\prime}=0$ on $\left[0, T_{0}\right] \cup\left[T_{1}, T\right]$ and we obtain

$$
\left\|\chi^{\prime} z\right\|_{L^{2}\left(0, T ; H_{0}^{1}(\Omega)\right)} \leq C\left\|\frac{\partial z}{\partial \nu}\right\|_{L^{2}\left((0, T) \times \Gamma_{0}\right)}
$$

with $C=C(\chi)$. Moreover, $w=\chi z$ satisfies :

$$
\left\{\begin{array}{l}
i w^{\prime}+\Delta w+q w=i \chi^{\prime} z, \quad \text { in } \Omega \times(0, T) \\
w=0, \quad \text { on } \partial \Omega \times(0, T) \\
w(T)=0, \quad \text { in } \Omega
\end{array}\right.
$$

Then, applying again Lemma 7 and since $\chi^{\prime} z \in L^{2}\left(0, T ; H_{0}^{1}(\Omega)\right)$, we have

$$
\|w(t)\|_{H_{0}^{1}(\Omega)} \leq C\left\|\chi^{\prime} z\right\|_{L^{2}\left(0, T ; H_{0}^{1}(\Omega)\right)}, \quad \forall t \in[0, T] .
$$

Therefore, with $t=0$ and recalling that $w(0)=z_{0}$, we obtain :

$$
\left\|z_{0}\right\|_{H_{0}^{1}(\Omega)} \leq C\left\|\chi^{\prime} z\right\|_{L^{2}\left(0, T ; H_{0}^{1}(\Omega)\right)} \leq C\left\|\frac{\partial z}{\partial \nu}\right\|_{L^{2}\left((0, T) \times \Gamma_{0}\right)},
$$

what proves the inverse inequality (18).

Thereafter, we manage to obtain better stability results about our inverse problem. The main idea is to use this observability estimate and the price to pay is to assume more regularity on the given function $R$ and to obtain a result with non explicit constants that we had till now. 
Theorem 11. Let $q \in L^{\infty}(\Omega)$ and $u$ be a solution of equation (2).

Assume that

$\Gamma_{0}$ satisfies (6),

$$
\begin{gathered}
R \in W^{1,2}\left(0, T, W^{1, \infty}(\Omega)\right) \cap W^{2,1}\left(0, T ; L^{\infty}(\Omega)\right), \\
R(0) \text { is real valued and }|R(x, 0)| \geq r_{0}>0 \text {, ae in } \bar{\Omega} .
\end{gathered}
$$

Then, there exists a constant $C=C\left(\Omega, T, \Gamma_{0},\|q\|_{L^{\infty}(\Omega)}, R\right)>0$ such that for all $f \in H_{0}^{1}(\Omega)$,

$$
C^{-1}\|f\|_{H_{0}^{1}(\Omega)} \leq\left\|\frac{\partial u}{\partial \nu}\right\|_{H^{1}\left(0, T ; L^{2}\left(\Gamma_{0}\right)\right)} \leq C\|f\|_{H_{0}^{1}(\Omega)} .
$$

\section{Proof :}

We work on equation (11), satisfied by $v=u^{\prime}$ :

$$
\left\{\begin{array}{l}
i v^{\prime}(x, t)+\Delta v(x, t)+q(x) v(x, t)=f(x) R^{\prime}(x, t), x \in \Omega, t \in(0, T) \\
v(x, t)=0, x \in \partial \Omega, t \in(0, T) \\
v(x, 0)=-i f(x) R(x, 0), x \in \Omega
\end{array}\right.
$$

We introduce :

$$
\begin{cases}i z^{\prime}+\Delta z+q z=0, & \text { in } \Omega \times(0, T) \\ z=0, & \text { on } \partial \Omega \times(0, T) \\ z(0)=-i f R(0), & \text { in } \Omega\end{cases}
$$

and

$$
\begin{cases}i \varphi^{\prime}+\Delta \varphi+q \varphi=f R^{\prime}, & \text { in } \Omega \times(0, T) \\ \varphi=0, & \text { on } \partial \Omega \times(0, T) \\ \varphi(0)=0, & \text { in } \Omega\end{cases}
$$

Then,

$$
v(x, t)=z(x, t)+\varphi(x, t), \quad x \in \Omega, t \in(0, T) .
$$

On the one hand, as we have $R \in W^{2,1}\left(0, T, L^{\infty}(\Omega)\right)$ and because of Lemma 7, we can write :

$$
\left\|\frac{\partial \varphi}{\partial \nu}\right\|_{L^{2}\left((0, T) \times \Gamma_{0}\right)} \leq C\left\|f R^{\prime}\right\|_{W^{1,1}\left(0, T ; L^{2}(\Omega)\right)} \leq C\|f\|_{L^{2}(\Omega)} .
$$

On the other hand, since $R \in W^{1,2}\left(0, T, W^{1, \infty}(\Omega)\right)$ the observability inequality gives

$$
\|f R(0)\|_{H_{0}^{1}(\Omega)} \leq C\left\|\frac{\partial z}{\partial \nu}\right\|_{L^{2}\left((0, T) \times \Gamma_{0}\right)} .
$$

Moreover, we have to notice that if $R(0) \in W^{1, \infty}(\Omega)$ and $|R(x, 0)| \geq r_{0}>0$, then $\frac{1}{R(0)} \in W^{1, \infty}(\Omega)$ and it yields

$$
\|f\|_{H_{0}^{1}(\Omega)} \leq C\|f R(0)\|_{H_{0}^{1}(\Omega)} .
$$


We finally obtain :

$$
\|f\|_{H_{0}^{1}(\Omega)} \leq C\left\|\frac{\partial v}{\partial \nu}\right\|_{L^{2}\left((0, T) \times \Gamma_{0}\right)}+C\|f\|_{L^{2}(\Omega)} .
$$

Then, writting this estimate with $u$ and applying Theorem 4 to take away the term $\|f\|_{L^{2}(\Omega)}$, we obtain the left hand side of (19).

To conclude, we can directly apply Lemma 7 to (11) since $f \in H_{0}^{1}(\Omega)$ and $R \in$ $W^{1,2}\left(0, T, W^{1, \infty}(\Omega)\right)$. We then obtain :

$$
\begin{aligned}
\left\|\frac{\partial v}{\partial \nu}\right\|_{L^{2}\left(0, T ; L^{2}\left(\Gamma_{0}\right)\right)} & \leq C\|f R(0)\|_{H_{0}^{1}(\Omega)}+C\left\|f R^{\prime}\right\|_{L^{1}\left(0, T ; H_{0}^{1}(\Omega)\right)} \\
& \leq C\|f\|_{H_{0}^{1}(\Omega)} .
\end{aligned}
$$

Since $v=u^{\prime}$, we actually know there exists a strictly positive constant $C$ depending on $\Omega, T, \Gamma_{0},\|q\|_{L^{\infty}(\Omega)}$ and $R$ such that

$$
C^{-1}\|f\|_{H_{0}^{1}(\Omega)} \leq\left\|\frac{\partial u}{\partial \nu}\right\|_{H^{1}\left(0, T ; L^{2}\left(\Gamma_{0}\right)\right)} \leq C\|f\|_{H_{0}^{1}(\Omega)}
$$

and the proof of Theorem 11 is complete.

As for the proof of Theorem 1 and Corollary 2 in Section 5, we can derive from Theorem 11 the following one.

Theorem 12. Let $\mathcal{U}$ be a bounded subset of $L^{\infty}(\Omega)$ and $q \in L^{\infty}(\Omega)$. Assume that

$\Gamma_{0}$ satisfies (6),

$$
\begin{gathered}
y(q) \in W^{1,2}\left(0, T, W^{1, \infty}(\Omega)\right) \cap W^{2,1}\left(0, T, L^{\infty}(\Omega)\right), \\
y_{0} \text { is real valued and }\left|y_{0}\right| \geq r_{0}>0, \text { ae in } \bar{\Omega} .
\end{gathered}
$$

Then, there exists a constant $C=C\left(\Omega, T, \Gamma_{0},\|q\|_{L^{\infty}}, y_{0}, h, \mathcal{U}\right)>0$ such that $\forall p \in$ $\mathcal{U}$ verifying $q-p \in H_{0}^{1}(\Omega)$

$$
C^{-1}\|p-q\|_{H_{0}^{1}(\Omega)} \leq\left\|\frac{\partial y(q)}{\partial \nu}-\frac{\partial y(p)}{\partial \nu}\right\|_{H^{1}\left(0, T ; L^{2}\left(\Gamma_{0}\right)\right)} \leq C\|p-q\|_{H_{0}^{1}(\Omega)} .
$$

\section{References}

[1] A. L. BukHGeIM, Introduction to the theory of Inverse Problems, Inverse and Ill-posed problem Series. VSP, Utrecht, 2000.

[2] A. L. BukHGEIM and G. UhLMAnn, Recovering a potential from partial Cauchy data, Comm. Partial Differential Equations 27 (2002), 653-668.

[3] R. DAutray and J.-L. LiOns, Mathematical analysis and numerical methods for science and technology, Springer, Berlin, 2000, Vol. 1 et 5. 
[4] V. Isakov, Inverse Problems for Partial Differencial Equations., Springer Verlag, Berlin, 1998.

[5] O. Yu. Imanuvilov and M. Yamamoto, Global uniqueness and stability in determining coefficients of wave equations., Comm. Partial Differential Equations 26 No 7-8 (2001), 1409-1425.

[6] O. Yu. IMANUVILOV and M. YAMAMOTO, Global lipschitz stability in an inverse hyperbolic problem by interior observations, Inverse Problems 17 No 4 (2001), 717-728.

[7] M.V. Klibanov, Inverse Problems and Carleman estimates, Inverse Problems 8 (1992), 575-596.

[8] I. LASIECKA et R. TRIGgIANI, Optimal regularity, exact controllability and uniform stabilization of the Schrödinger equations with Dirichlet control, Differential and Integral Equations, 5 No 3 (1992), 521-535.

[9] E. MACHTYNGIER, Exact controllability for the Schrödinger equation, SIAM J. Control and Optimization 32 No 1 (1994), 24-34.

[10] J.-P. Puel and M. Yамамото, Smoothing property in multidimentional inverse hyperbolic problems : application to uniqueness and stability., J of Inverse and Ill-posed Problems 4 (1996), 283-296.

[11] R. TRIGgiani, Carleman estimates and exact boundary controllability for a system of coupled non-conservative Schrödinger equations, Special issue Rend. Istit. Mat. Univ. Trieste, XXVIII (1997), 453-504. Supplement, Dedicated to the memory of Pierre Grisvard.

[12] R. TRIGGIANI and P.- F. YAO, Inverse estimates for Schrödinger equations with variable coefficients, Control and Cybernetics, 28 No 3 (1999), 627-664.

[13] М. Yамамото, Uniqueness and stability in multidimensional hyperbolic inverse problems, J. Math. Pures Appl. 78 (1999), 65-98. 